\title{
Ebisu Ebisu
}

Études japonaises Études japonaises

$53 \mid 2016$

1914-1918, une guerre mondiale ? La perspective japonaise

\section{À l'aube d'un siècle Pacifique : les États-Unis et le Japon durant la Première Guerre mondiale \\ アジア太平洋時代の黎明期一第一次世界大戦における日本とアメリ \\ 力}

Dawn of a Pacific Century: The U.S. and Japan in World War I

\section{Frederick R. Dickinson}

Traducteur : Jean-Philippe Miller-Tremblay

\section{OpenEdition}

\section{Journals}

Édition électronique

URL : https://journals.openedition.org/ebisu/1853

DOI : 10.4000/ebisu. 1853

ISSN : 2189-1893

\section{Éditeur}

Institut français de recherche sur le Japon à la Maison franco-japonaise (UMIFRE 19 MEAE-CNRS)

\section{Édition imprimée}

Date de publication : 10 décembre 2016

Pagination : 77-100

ISSN : 1340-3656

\section{Référence électronique}

Frederick R. Dickinson, «À l'aube d'un siècle Pacifique : les États-Unis et le Japon durant la Première Guerre mondiale », Ebisu [En ligne], 53 | 2016, mis en ligne le 10 décembre 2016, consulté le 08 novembre 2021. URL : http://journals.openedition.org/ebisu/1853; DOI : https://doi.org/10.4000/ ebisu. 1853 


\section{À l'aube d'un siècle Pacifique}

Les États-Unis et le Japon

durant la Première Guerre mondiale

Frederick R. DickINson

アジア太平洋時代の黎明期

第一次世界大戦における日本とアメリカ

フレデリック・R・ディキンソン

Dawn of a Pacific Century:

The U.S. and Japan in World War I

Frederick R. Dickinson

\begin{abstract}
\ots-clés : Première Guerre mondiale, Japon, Asie-Pacifique, commerce, réformes.
\end{abstract}

L'auteur : Frederick R. Dickinson est professeur en histoire japonaise et codirecteur du Lauder Institute of Management and International Studies à l'université de Pennsylvanie. Il est l'auteur de World War I and the Triumph of a New Japan, 1919-1930 (2013) entre autres publications.

Résumé : Les historiens des relations entre le Japon et les États-Unis décrivent habituellement la Première Guerre mondiale comme une période de tensions croissantes ayant mené à la guerre du Pacifique. Mais le repli des puissances européennes sur elles-mêmes entre 1914 et 1918 a favorisé le resserrement des liens économiques entre les deux pays, tandis que la participation active du Japon à la nouvelle paix mondiale menée par les Américains après 1918 a renforcé leur coopération politique durant les années 1920. Vue depuis 1941, la Première Guerre mondiale peut apparaître comme un simple prélude à la Seconde, mais depuis une perspective contemporaine, les années 1910 et 1920 marquent la fondation institutionnelle d'un nouvel ordre mondial centré sur l'Asie-Pacifique. 
キーワード
第一次世界大戦、日本、アジア／太平洋、 貿易、改革

\section{著者}

フレドリック・R・デイキンソンはペンシル ベニア大学日本史教授兼ローダー研究所副理 事。著書に『World War I and the Triumph of a New Japan, 1919-1930』(2013) がある。

\section{要旨}

日米関係史の専門家は、第一次世界大戦を双 方の摩擦が大きくなった時期、つまり、太平 洋戦争の基礎と見なしている。しかし、1914 年- 18 年の間、ヨーロッパ勢力の低下に伴い、 日米経済関係が深化していく。そして 20 年 代において、日本がアメリカの建設した世界 平和に積極的に参加することによって、双方 の政治的協力も深まっていく。1941 年から 見た第一次世界大戦は第二次世界大戦の前奏 曲としてしか見られない。しかし、私達の時 代から眺めれば、1910 年- 20 年代はアジア 太平洋を中心とした新しい世界の基盤だった。

War as an era of growing bilateral tensions that ultimately laid the foundation for the Pacific War. But the inward turn of European power between 1914 and 1918 helped deepen US-Japan economic ties. And Japan's active participation in the new American-led peace after 1918 intensified bilateral political cooperation through the 1920s. From the vantage point of 1941, the First World War looks like nothing more than a prelude to World War II. From the perspective of our own era, however, the 1910s and 20s mark the institutional foundation for a new Asia-Pacific world.

Abstract: Historians of US-Japan relations typically describe the First World 


\title{
À l'aube d'un siècle Pacifique Les États-Unis et le Japon durant la Première Guerre mondiale
}

Frederick R. DICKINSON*

\begin{abstract}
"Le chemin parcouru par le Japon ces cinquante dernières années n'a pas d'égal dans l'histoire du monde. Le Japon a joué un rôle extraordinairement utile pour la cause alliée dans cette guerre pour la civilisation. "

Theodore Roosevelt, 30 novembre $1919^{1}$
\end{abstract}

Les chercheurs qui ont étudié la Première Guerre mondiale ont souvent tenté de dégager les causes qui expliqueraient la Seconde Guerre mondiale. L'étude classique de Fritz Fischer sur l'Allemagne durant la Grande Guerre fit trembler le monde académique précisément parce qu'il y démontrait que les objectifs de guerre allemands de 1914 agissaient encore en 1939 (Fischer 1961). Même si les chercheurs japonais ont tardé à considérer cette catastrophe inaugurale qu'est la Première Guerre mondiale comme un des tournants décisifs $\mathrm{du} \mathrm{xx}^{\mathrm{e}}$ siècle, des études récentes en langue japonaise ont à leur tour exploré de telles causes, se faisant l'écho des préoccupations occidentales d'après-1945. Yamamuro Shin.ichi a décrit la Grande Guerre comme une «ligne de fracture " entre le monde du XIx ${ }^{\mathrm{e}}$ siècle et

1. Theodore Roosevelt, "What the Japanese stood for in the World War ", New York Times, 30 nov. 1919, réimprimé dans Roosevelt (1920). L'accès à ce document a été rendu possible grâce à la bibliothèque de la faculté de droit de l'université de Kyoto.

* University of Pennsylvania. 
une nouvelle ère de guerre totale (Yamamuro 2011) ${ }^{2}$. Les historiens des relations entre les États-Unis et le Japon ont longtemps élevé cette guerre au rang de référence pour la considérer comme un tournant dans l'évolution d'un rapport bilatéral relativement amical vers une ère de ténèbres et, en définitive, vers la guerre ${ }^{3}$.

En effet, le récit canonique des liens entre les États-Unis et le Japon au début du $\mathrm{xx}^{\mathrm{e}}$ siècle met en évidence des tensions latentes remontant au moins à la guerre russo-japonaise. Le président américain Theodore Roosevelt est fréquemment cité pour avoir affirmé à Kaneko Kentarō 金子 堅太郎 (1853-1942), son ancien camarade à Harvard, que la victoire japonaise contre la flotte russe de la Baltique durant la bataille de Tsushima en mai 1905 fut "le plus grand prodige auquel le monde ait assisté " (Okamoto 1970 : 119). Mais ces propos célébrant le succès japonais sont fréquemment nuancés par une déclaration antérieure d'un Roosevelt affirmant sur un ton moqueur: "Je suis parfaitement conscient que s'ils [les Japonais] gagnent [la guerre russo-japonaise], cela signifiera peut-être une lutte future entre eux et nous " (Esthus 1967 : 43). En effet, seulement deux ans après la guerre, Roosevelt déploya la Grande flotte blanche dans les eaux japonaises, en réponse à une légère pointe d'agressivité détectée dans les communications japonaises (Griswold 1938 : 127). Au même moment, la présence grandissante de ressortissants japonais en Californie donna naissance à une série de mesures locales et nationales en vue de restreindre ce flux migratoire, débutant en 1906 avec la décision du conseil local de l'éducation d'interdire l'accès aux écoles publiques de San Francisco à 93 étudiants nippo-américains et coréano-américains ${ }^{4}$. Avant 1909, la nouvelle

2. De la même façon, Naraoka Sōchi a récemment ressuscité la vieille idée selon laquelle les Vingt-et-une demandes de 1915 marquèrent le début d'un antagonisme sinojaponais qui mènera finalement à une guerre d'envergure en 1937 (Naraoka 2015). J'avais déjà remis en question le lien entre les Vingt-et-une demandes et l'expansion japonaise durant les années 1930 dans Dickinson (1999).

3. Voir l'étude classique de Griswold (1938 : chap. 5), et plus récemment, Kawamura (2000). Pour une exception notable, voir Kane (2010).

4. Cet évènement fut suivi de l'entente amiable par laquelle le Japon accepta de restreindre l'émigration vers les États-Unis (Gentleman's Agreement de 1907). En 1913, la Loi de l'État de Californie sur l'accession à la propriété immobilière pour les étrangers (California's Alien Land Law) interdit aux "étrangers n'ayant pas droit à la citoyenneté " de posséder des terres agricoles ou des baux à long terme sur ces mêmes terres. Cette 
administration d'Howard Taft tenta de mettre un frein à la croissance de la puissance japonaise à proximité de l'archipel. Même si, en définitive, une grande force de résistance contrecarra ce projet, la stratégie adoptée par Taft pour "neutraliser » l'expansion économique et politique japonaise en Mandchourie représentait la rivalité géopolitique croissante entre les ÉtatsUnis et le Japon en Asie (LaFeber 1997 : chap. 3).

\section{La Première Guerre mondiale entre les États-Unis et le Japon}

À la lumière de ces épisodes de rivalité, le déclenchement de la guerre en août 1914 créa naturellement de nouvelles opportunités pour une compétition trans-pacifique. Mais contrairement à la question de l'immigration en Californie ou au projet de neutralisation en Mandchourie, les tensions entre les États-Unis et le Japon durant la Grande Guerre étaient moins fondées sur des griefs concrets que sur de vagues angoisses concernant de profondes transformations géopolitiques. Entre 1914 et 1918, les ÉtatsUnis comme le Japon avaient d'autres préoccupations plus immédiates que celles de se lancer dans des négociations au sujet des relations bilatérales.

Le récit canonique des liens entre les États-Unis et le Japon durant la Première Guerre mondiale met l'accent sur la tension provoquée par l'expansion du pouvoir japonais en Chine (les Vingt-et-une demandes, les prêts américains à la Chine et l'accord Lansing-Ishii), sur l'ampleur surprenante de la participation japonaise à l'expédition de Sibérie en 1918, ainsi que sur l'agitation liée à la proposition d'introduire le principe d' "égalité des races » et aux revendications de Tokyo sur la province chinoise du Shandong pendant la conférence de paix à Paris en $1919^{5}$. Selon la vision

loi visait les citoyens japonais (ainsi que les citoyens chinois, indiens et coréens). Sur ce conflit, ainsi que sur son legs durant l'entre-deux-guerres, voir Hirobe (2001).

5. Sur les Vingt-et-une demandes, voir Griswold (1938) et Takahara (2006b: chap. 1). Sur les prêts américains, voir Israel (1971 : chap. 5). Sur l'accord Lansing-Ishii, voir Beers (1962) et Takahara (1997). Takahara attribue à cet accord le mérite d'avoir amélioré les relations entre les États-Unis et le Japon. Sur l'expédition de Sibérie, voir Morley (1957), Takahara (2006a) et Richard (2013). Il est intéressant de constater que tandis que Richard explique la présence prolongée des États-Unis en Sibérie (une année et demie après l'armistice) par une peur de l'expansion japonaise, il soutient que Wilson, 
classique des relations entre les États-Unis et l'Asie orientale, "l'antagonisme larvé entre les Japonais et les Américains atteignit un point culminant durant la Première Guerre mondiale» (Iriye 1992 : 131).

Sans nier la réalité de ces tensions bilatérales, il est nécessaire de noter que ce récit est particulièrement utile pour expliquer rétroactivement Pearl Harbor. Cependant, plus la distance chronologique avec la Seconde Guerre mondiale est grande, plus il nous faut admettre que les relations entre les États-Unis et le Japon ne représentaient pas l'essentiel des préoccupations des décideurs de part et d'autre entre 1914 et 1918. Au contraire, le déclenchement de la Première Guerre mondiale montre que Tokyo et Washington avaient des centres d'intérêts fortement divergents. D'un côté, durant son premier mandat (1913-17), la plus grande partie des efforts au niveau géopolitique du président américain Woodrow Wilson visèrent non seulement à maintenir les États-Unis hors de la Première Guerre mondiale mais également à conclure un accord en Europe (Kennedy 2009). De l'autre, le cabinet japonais décida d'entrer en guerre contre l'Allemagne le 8 août 1914, seulement quatre jours après la déclaration de guerre officielle de son allié, la Grande-Bretagne (Dickinson 2003).

Il y eut, dès le déclenchement des hostilités, un grand nombre de voix qui s'élevèrent des deux côtés de l'océan Pacifique, anticipant les conséquences défavorables de ce conflit sur les relations entre les États-Unis et le Japon. Dans un mémorandum daté du mois d'août 1914 et destiné au Cabinet, le maréchal Yamagata Aritomo 山縣有朋 (1838-1922) commenta l'ascension rapide des États-Unis au rang de puissance mondiale ainsi que ses intérêts grandissants en Asie. Les États-Unis avaient montré une résistance des plus acharnées à la progression japonaise en Mandchourie du Sud, tandis que l'attention européenne se déplaçait vers l'Europe durant la guerre, accentuant ainsi la puissance potentielle des États-Unis en Chine. Selon Yamagata, non seulement la guerre n'aurait aucun effet négatif sur ce simple spectateur, mais celui-ci pourrait également s'emparer d'un

au commencement de cette expédition, se préoccupait plutôt de la Grande-Bretagne et de la France que du Japon. Sur le principe d'" égalité des races ", voir Shimazu (1998). Sur le Shandong, voir Fifield (1965), Nishida (2002) et Elleman (2002). Il faut remarquer qu'Elleman avance - contre la doxa - l'argument selon lequel Wilson, à Paris, négocia avec succès un compromis entre le Japon et la Chine sur le Shandong. 
" monopole sur la récompense " que serait la Chine ${ }^{6}$. Au même moment, face à de nombreuses récriminations dans la presse américaine, le premier ministre japonais Ōkuma Shigenobu 大隈重信 (1838-1922) se sentit contraint, le jour suivant la déclaration de guerre, de clarifier ses intentions dans un télégramme envoyé au New York Independent. Intitulée «Message to the American people», cette déclaration garantissait aux lecteurs que "le Japon n'a aucune intention cachée, aucun désir de sécuriser de nouveaux territoires [et] n'envisage pas de priver la Chine ou tout autre peuple de ce qu'ils possèdent actuellement ${ }^{7}{ }^{\prime}$.

\section{L'effort de guerre allié et le Japon}

Même si le Japon agit rapidement lorsqu'il déclara la guerre à l'Allemagne - cette action visant à saisir l'opportunité d'étendre considérablement son autorité sur la région Asie-Pacifique - il contribua de manière décisive à l'effort de guerre allié jusqu'en 1918. Déjà en septembre 1914, deux forces opérationnelles de la Marine impériale repoussèrent les navires de l'escadre allemande d'Extrême-Orient hors du Pacifique Sud, assurant au Japon non seulement l'augmentation de son pouvoir en Micronésie allemande (les îles Marshall, Mariannes et Carolines), mais permettant également le déploiement d'une présence australienne, néo-zélandaise et britannique dans les possessions allemandes de Nouvelle-Guinée et de Samoa ${ }^{8}$. L'assaut victorieux contre la forteresse allemande de Qingdao en Chine en novembre 1914, mené par 29000 soldats japonais et 2800 soldats des troupes impériales britanniques, allait faire du Japon et de la GrandeBretagne les deux plus importants acteurs politiques et économiques en Chine durant les années $1930^{9}$.

6. Yamagata Aritomo, “Mon avis sur la politique en Chine " (Shina iken 支那意見), in Ōyama (1966: 345).

7. Cité dans Elleman (2002: 12).

8. Après la guerre, tous ces anciens territoires furent partagés entre le Japon, l'Australie, la Nouvelle-Zélande et la Grande-Bretagne et furent considérés comme des mandats de classe C par la Société des Nations nouvellement constituée.

9. Ces effectifs sont issus de Eguchi (1989 : 20). 
L'affrontement entre le Japon et l'Allemagne en Asie se termina en novembre 1914. Mais son effort de guerre auprès des Alliés se poursuivit jusqu'en 1918. Une flotte composée de quatre navires de combat appartenant à la $1^{\text {re }}$ escadre japonaise protégea les routes de navigation dans l'océan Pacifique à partir de septembre $1914^{10}$. Durant le printemps 1915, une autre flotte de trois navires se joignit au groupe de combat de la Marine royale britannique patrouillant la côte ouest américaine, du canal de Panama jusqu'au Canada ${ }^{11}$. Entre 1914 et 1918 , des navires de la $3^{\text {e }}$ flotte japonaise escortèrent des troupes australiennes et néo-zélandaises de l'océan Pacifique vers Aden en mer d'Arabie, en passant par l'océan Indien. En février 1915, des marins japonais se joignirent à des marins britanniques et français pour mettre fin à une révolte de soldats indiens à Singapour ${ }^{12}$. Suite à plusieurs attaques contre des vaisseaux marchands japonais, trois divisions de contre-torpilleurs japonais et un cuirassé participèrent aux côtés des Alliés à l'affrontement contre des sous-marins allemands en mer Méditerranée en février $1917^{13}$.

Là où la puissance militaire japonaise n'était pas directement impliquée, une aide substantielle était acheminée. Plusieurs unités de la Croix-Rouge japonaise opérèrent dans les capitales alliées durant la guerre (Araki 2014). Le trafic maritime japonais dans les eaux européennes entre 1914 et 1918 s'éleva à 200000 tonnes (Togo 1918 : 373). En avril 1917, 311 vaisseaux japonais furent affrétés par des étrangers pour contribuer au commerce de guerre en général ${ }^{14}$. Durant la guerre, des navires japonais affrétés transportèrent 100000 tonnes de charbon et de ravitaillements entre la GrandeBretagne et la France (Togo 1918 : 373). De plus, le Japon fournit du cuivre et des devises (dont 640 millions de yens en prêts) aux alliés qui en

10. Hashiguchi Jihei, "Japan's Share in the Naval Operations of the Great War ", Japan Dept. of the Navy, 8 déc. 1918, p. 4 (WDGS/WDSS, Folder: 003011-026-0568).

11. Ibid., p. 5.

12. Ibid., p. 3.

13. Lettre d'Edwin Denby, secrétaire à la Marine, au secrétaire d'État américain, 23 septembre 1921 (SKHP, Box 255, "Japan: War Costs and Contributions » file).

14. "Shipping and Shipbuilding Industry of Japan", 19 mars 1918, p. 8 (WDGS/ WDSS, Folder: 003011-026-0568-0001). 
avaient grand besoin ${ }^{15}$. À la Russie furent vendus 600000 fusils et transférés trois cuirassés japonais ${ }^{16}$. Pour la France, le Japon construisit douze contre-torpilleurs, livrés directement en mer Méditerranée ${ }^{17}$.

\section{L'effort de guerre américain et le Japon}

Les contemporains américains reconnurent clairement la valeur des contributions japonaises à la guerre. En 1918, Payson Jackson Treat, professeur d'histoire à l'université de Stanford, déclara que sans l'aide militaire et financière japonaise, "l'effondrement de la Russie se serait produit bien avant ". Dans une plus sombre perspective, il ajouta que si le Japon avait pris la décision de rejoindre les Empires centraux, la « Russie aurait eu à mobiliser une grande armée en Extrême-Orient, les populations des colonies britanniques n'auraient sans doute pas osé quitter leurs propres territoires, et le contrôle sur le commerce dans le Pacifique aurait échappé aux Alliés " (Treat 1918 : 7). Theodore Roosevelt reconnut également que sans la présence militaire japonaise en Asie-Pacifique «il est probable qu'en ce moment une force américano-britannique assiègerait Kiao-Chao [Jiaozhou] et que notre commerce souffrirait des raids allemands dans le Pacifique» (Roosevelt 1920 : 24). Les planificateurs militaires américains furent à tel point sensibles à l'importance décisive de l'aide japonaise que la Marine américaine déclara en juillet 1918 qu’il était « de nécessité vitale » d'obtenir l'aide des cuirassés japonais pour protéger les transports de troupes américaines lors de la traversée de l'océan Atlantique ${ }^{18}$.

15. Lieutenant-colonel K. F. Baldwin, bureau du Chef d'état-major, département de la Guerre, division du Renseignement militaire, "A Brief Account of Japan's Part in the World War", 16 sept. 1921, p. 6 (SKHP, Box 255, "Japan: War Costs and Contributions»). Si nous incluons les prêts à la Chine, nous atteignons la somme approximative d'un milliard de yens. Entre 1916 et 1918, le Japon prêta à la Chine 280 millions de yens, provenant de sources publiques et privées (Asher 2002 : 73).

16. Ces cuirassés avaient été capturés lors de la guerre russo-japonaise : le Sagami (le nom original étant Peresvet), le Tango (Poltava), et le Soya (Varyag); voir Roosevelt (1920 : 25). L'information sur les fusils est tirée du compte rendu de K. F. Baldwin, p. 6 (voir note 15).

17. Compte rendu de Hashiguchi Jihei, p. 1 (voir note 10).

18. Lettre d'Edwin Denby (voir note 13). 
Toutefois, il y eut une conséquence encore plus vitale sur le long terme pour les États-Unis et leurs nouvelles relations bilatérales avec le Japon : l'interdépendance économique. Après la guerre, leurs deux économies émergèrent comme les principales bénéficiaires de la déflagration européenne. En fait, la Grande Guerre provoqua une transformation économique majeure dans ces deux pays. D'un côté, elle marqua pour le Japon la transition d'une économie agraire vers une économie industrielle (Hayami \& Kojima 2004 : 226-33). De l'autre, les États-Unis devinrent, après la guerre, le principal centre de la production industrielle, de la finance et du commerce mondial, surpassant ainsi l'Europe de l'Ouest (Frieden 2007 : 132). Ces changements spectaculaires au niveau économique des deux côtés du Pacifique soulevèrent nécessairement de nouvelles interrogations sur un possible renouvellement des tensions bilatérales. Les récits canoniques des relations entre les États-Unis et le Japon ont accentué ces préoccupations. Suetake Yoshiya a mis en évidence une vague de commentaires au Japon à partir de 1917, définissant l'après-guerre comme une nouvelle ère de conflit économique (Suetake 1998).

Mais, durant la guerre, la dépendance des États-Unis envers la robuste économie japonaise atteignit un niveau remarquable. En conservant leur neutralité jusqu'en avril 1917, les États-Unis, comme nous l'avons constaté, comptèrent sur les marines japonaise et britannique pour maintenir la sécurité des zones côtières américaines dans l'océan Pacifique. Considérant l'énorme profit potentiel du commerce atlantique, les États-Unis devinrent également et immédiatement redevables au Japon dans toute la région Pacifique. À la recherche de profits du côté atlantique, toutes les principales compagnies maritimes américaines s'y déplacèrent peu après le déclenchement des hostilités. Les compagnies japonaises en étaient venues à contrôler, en 1917, plus de $55 \%$ du transport de marchandises dans le Pacifique, tandis que les compagnies américaines n'en contrôlaient que 2\% (Safford 1970 : 440). Une bonne partie de ce commerce fut consacrée à maintenir les moyens de subsistance du Japon. Mais les cargos japonais transportèrent également des céréales, de l'Australie et de l'Amérique du Sud vers l'Europe. De plus, le commerce direct avec les États-Unis dépassa les 333 millions de dollars durant l'année fiscale prenant fin en juin 1917, les exportations japonaises vers les États-Unis correspondant à la plus grande partie de ce montant (203 millions de dollars; Togo 1918 : 375). 
Ces incroyables prouesses commerciales japonaises furent rendues possibles par une énorme augmentation de la production navale. Entre le déclenchement de la guerre et l'été 1917, les 29 constructeurs japonais produisirent l'équivalent de 600000 tonnes de navires (Togo 1918 : 373). Une grande quantité de tôles d'acier, de tôles de chaudronnerie, de barres d'acier et d'autres matériaux produits dans les fonderies américaines furent nécessaires pour leur construction ${ }^{19}$. De son côté, le Japon devint un important fournisseur de produits industriels finis pour les États-Unis. Malgré leur formidable industrie de construction navale, les États-Unis signèrent un contrat avec le Japon afin d'affréter l'équivalent de 150000 tonnes de navires pour les utiliser, en Atlantique, dans le commerce de guerre. Au même moment, une entente fut conclue pour l'achat de 45 navires de charge japonais (Safford 1970 : 448-449). Deux mois plus tard, l'attaché de la Marine américaine négocia un achat de platine à Tokyo, pour une valeur de 100000 yens $^{20}$. Dès juin 1917, le capitaine William V. Pratt prit note d'une nouvelle attitude de collaboration entre les États-Unis et le Japon qui, affirma-t-il, réduisait le risque d'hostilités entre les deux nations ${ }^{21}$.

Pratt venait tout juste de reconnaitre ce nouvel esprit de coopération bilatérale quand, en juillet 1917, les États-Unis déclarèrent un embargo sur les ventes d'acier au Japon, ce qui souleva un tollé à Tokyo. Comme l'a noté Jeffrey Safford, cet embargo déclencha immédiatement une mission diplomatique japonaise de haut niveau aux États-Unis, dont le résultat fut l'accord Lansing-Ishii en novembre $1917^{22}$. Conjointement à la réaction de Washington aux prêts consentis par des consortiums à la Chine, aux Vingt-et-une demandes et à l'afflux de troupes japonaises en Mandchourie

19. En juin 1917, le Japon avait déjà commandé aux États-Unis 208000 tonnes de tôles d'acier, 5200 tonnes de tôles de chaudronnerie, 4200 tonnes de barres d'acier et 120000 tonnes d'autres matériaux en acier ("Shipping and Shipbuilding Industry of Japan ", p. 3 ; voir note 14).

20. Télégramme du major K. F. Baldwin (Tokyo) au département de la Guerre, 22 mai 1918, p. 1 (WDGS/WDSS, Folder: 003011-001-0002).

21. Williams (1996 : 23). Pratt était membre de l'état-major de l'amiral William S. Benson, chef des opérations navales américaines.

22. Le troisième assistant au secrétaire d'État, Breckinridge Long, qui accueillit Ishii à San Francisco, fit parvenir un télégramme au département d'État expliquant que l'acier était un sujet d'importance capitale pour la délégation japonaise (Safford 1970 : 443). 
et en Sibérie durant l'expédition homonyme, cet embargo a été décrit comme une tentative de «limiter les ambitions de l'Empire japonais dans le Pacifique pendant les années de guerre " (Safford 1970 : 439).

Mais au moment même où la formidable expansion maritime du Japon en temps de guerre préoccupait sérieusement Washington, des voix en nombre égal, sinon plus grand, insistaient sur l'importance de s'assurer l'appui du transport maritime japonais en vue du nouvel effort de guerre américain. Le président du Comité administratif pour le contrôle des exportations, Vance McCormick, informa le vicomte Ishii Kikujirō 石井 菊次郎 (1866-1945) en novembre 1917 que les États-Unis avaient l'intention d'obtenir le soutien du transport maritime japonais en usant de leur puissance économique si nécessaire. L'acier américain ne serait livré au Japon qu'en échange d'un envoi immédiat de navires aux États-Unis (Safford 1970 : 445). Loin d'être une tentative d'endiguement, l'embargo américain sur l'acier, qui se poursuivit pendant un an jusqu'en juillet 1918, visait à obtenir du Japon le plus grand soutien possible de son transport maritime. Ce fut, en d'autres mots, un autre signe de la nouvelle et considérable dépendance des États-Unis envers ses échanges commerciaux avec le Japon. Commentant la nouvelle entente de mars 1918 pour la location et l'achat de navires japonais, le capitaine K. F. Baldwin, attaché militaire américain à Tokyo, remarqua avec satisfaction que «les Japonais se sont montrés tout à fait disposés à aider les alliés et, dans cette optique, de vrais sacrifices ont été consentis par le gouvernement et par les usines ${ }^{23}$ ".

\section{L'héritage global de la Première Guerre mondiale au plan économique}

Malgré la surreprésentation, dans l'historiographie, de l'aspect conflictuel des relations entre les États-Unis et le Japon durant la Première Guerre mondiale, ce passé d'interdépendance économique en temps de guerre forma, après 1918, la base d'une nouvelle relation bilatérale sans précédent qui eut pour principale conséquence de déplacer le centre de gravité de

23. K. F. Baldwin, « Political Condition of Japan to Date », 9 avril 1918, p. 2 (WDGS/ WDSS, Folder: 003011-001-0002). 
l'économie globale. Malgré le bref embargo américain sur l'acier, le Japon doubla sa production de navires en 1918 par rapport à l'année précédente (Safford 1970 : 451). Les exportations de textiles japonais avaient presque triplé en 1919, tandis qu'en prenant pour point de départ l'année 1914, la valeur de toutes les marchandises expédiées à l'étranger avait plus que triplé2 ${ }^{24}$. Entre 1914 et 1919 , la valeur totale des exportations japonaises avait presque quadruplée ${ }^{25}$.

À l'échelle globale, ce développement remarquable de l'économie japonaise durant la guerre eut une conséquence des plus importantes : celle de propulser le commerce dans la région Asie-Pacifique à un tout nouveau rang au niveau mondial. La puissance économique florissante du Japon fut accompagnée par un déclin analogue de l'importance de l'économie européenne en Asie. Les exportations japonaises vers la Chine atteignirent 334 millions de yens en 1922, dépassant largement ses exportations vers la France (79 millions de yens) et la Grande-Bretagne (54 millions de yens) (Matsudaira 1925 : 409). Entre 1910 et 1920, le nombre de broches à coton en Chine appartenant à des Japonais bondit de 55296 à 801 662, dépassant largement les 256284 broches possédées par des intérêts occidentaux après la guerre (McClain 2002 : 396).

La proportion du commerce international dominée par les États-Unis et le Japon, à ce moment, était un phénomène encore plus frappant. Jusqu'en 1914, le Japon avait dû compter sur la Grande-Bretagne pour obtenir la plus grande partie de sa machinerie et de ses marchandises. Entre 1914 et 1939 par contre, l'Oncle Sam prit la première place parmi ses fournisseurs $^{26}$. Dès 1918, l'attaché militaire américain à Tokyo observa que " les Britanniques sentent que la situation favorable, au niveau commercial, des États-Unis au Japon et la confiance accordée par les Japonais aux

24. De 372,7 millions à 1,099 milliard de yens, et de 571,7 millions à 1,886 milliard de yens, respectivement (Ohkawa \& Shinohara 1979 : 316, tableau A26).

25. De 768 millions à 2,896 milliards de yens (Ohkawa \& Shinohara 1979: 334, tableau A31).

26. Beasley (1987 : 127, 211, tableaux 3 et 9, respectivement). Le Japon importait des États-Unis du fer, de l'acier, des moteurs, d'autres types de machineries, du pétrole, du coton et d'autres matières premières. 
États-Unis, tendent à placer l'influence britannique au second rang ${ }^{27}$ ". En 1922, le Japon importa des marchandises des États-Unis pour une valeur de 218,4 millions de dollars, soit presque 30 millions de plus que ce qui fut importé par la Chine, les Philippines, les Indes orientales néerlandaises, l'Indochine française, l'Inde britannique et le Siam réunis ${ }^{28}$.

Pour ce qui est des exportations, les États-Unis dépassèrent leurs concurrents durant les années 1890 grâce à l'achat de soie japonaise (Beasley 1987 : 127). Mais la part des États-Unis dans les exportations japonaises avait grimpé en 1922 à 733 millions de yens, surpassant de 266 millions de yens la valeur des exportations japonaises en Chine, en France et en GrandeBretagne réunies ${ }^{29}$. Tandis que le volume total du commerce japonais avec les États-Unis dépassait 333 millions de dollars en 1917, ce chiffre avait presque doublé en 1924, atteignant 600 millions, situant le Japon devant la France et l'Allemagne, et seulement derrière la Grande-Bretagne et le Canada, dans le commerce étranger américain (Matsudaira 1925 : 408).

\section{L'héritage global de la Première Guerre mondiale au plan politique}

Si la Grande Guerre a forgé de solides liens économiques entre les ÉtatsUnis et le Japon, elle créa également de nouvelles synergies politiques liant ces deux nations comme jamais auparavant. Tout comme le plus important partenaire économique externe du Japon ne fut plus la Grande-Bretagne mais bien les États-Unis, le même phénomène se produisit dans la sphère politique. Malgré le rôle majeur qu'avaient eu les États-Unis durant les années 1850 pour favoriser l'intégration du Japon dans le monde moderne $e^{30}$,

27. Lieutenant-colonel K. F. Baldwin, "British Efforts in Japan ", 9 déc. 1918, p. 1 (WDGS/WDSS, Folder: 003011-001-0002).

28. La valeur combinée des marchandises importées par ces territoires asiatiques s'élevait à 190,8 millions de dollars en 1922 (Matsudaira 1925 : 408).

29. Pour cette année, les exportations japonaises vers la Chine s'élevaient à 334 millions de yens tandis qu'elles étaient de 79 millions de yens pour la France et de 54 millions de yens pour la Grande-Bretagne (Matsudaira 1925 : 409).

30. Avec la guerre de l'Opium (1840-1841), la Grande-Bretagne et la France prirent les devants en introduisant un système dynamique de commerce extérieur avec la Chine. 
la Grande-Bretagne possédait une puissance militaire et économique supérieure qui lui assurait une autorité économique et politique incontestée sur le Japon de la fin du XIX ${ }^{\mathrm{e}}$ siècle. Tout comme les fondateurs du Japon moderne dépendirent du financement et des ingénieurs britanniques dans la construction d'un État moderne, ils construisirent une monarchie constitutionnelle et un système parlementaire rappelant le succès politique britannique ${ }^{31}$. Même les plus farouches partisans d'une réforme politique au Japon - bien que considérés par les fondateurs du Japon moderne comme une épine dans le pied - se tournaient vers Londres comme source principale d'inspiration. Leur objectif premier, au début $\mathrm{du} \mathrm{xx}^{\mathrm{e}}$ siècle, était moins d'en arriver à une "démocratie " à l'américaine que de perfectionner un "gouvernement constitutionnel" de type britannique ${ }^{32}$.

Toutefois, dès que les États-Unis prirent part à la Première Guerre mondiale, leur pouvoir politique à l'échelle globale apparut clairement. En janvier 1916, dans un appel décisif à la «réalisation " d'un gouvernement constitutionnel, Yoshino Sakuzō 吉野作造 (1878-1933), professeur à l'université impériale de Tokyo, soutint que le système politique idéal devait refléter une structure reposant sur le bipartisme, structure rencontrée en Grande-Bretagne comme aux États-Unis (Yoshino 1916a). Au même moment, l'« individualisme» (kojinshugi 個人主義) britannique et français demeurait au centre de ses préoccupations, comme la meilleure source d'inspiration pour le futur du Japon (Yoshino 1916b : 13). Toutefois, l'entrée en guerre des États-Unis en avril 1917 le poussa à se détourner totalement de ce point de référence. Il reconnut que la belligérance américaine ferait en sorte que l'opinion publique américaine " aurait un poids substantiel » dans tout accord, une fois la guerre terminée. Mais il fut authentiquement inspiré par la vision d'une nouvelle culture mondiale proposée par le président Woodrow Wilson. Cette culture, selon la description de Yoshino, avait pour principe le respect des droits des plus petites nations, pour objectif la paix

En revanche, les États-Unis jouèrent ce rôle auprès du Japon avec l'arrivée du commodore Perry dans la baie d'Uraga en 1853.

31. Sur l'importance politique de la Grande-Bretagne pour le Japon du XIX ${ }^{\mathrm{e}}$ siècle, voir Itō (1999) et Takii (2014).

32. Au début du $\mathrm{Xx}^{\mathrm{e}}$ siècle, les mouvements de réforme les plus puissants au Japon sont connus sous les noms de premier (1913) et second (1924) mouvement pour la protection d'un gouvernement constitutionnel (Kensei yōgo undō 憲政擁護運動). 
grâce à une association internationale rassemblant les États, et visait l'éradication de l'autocratie militaire (gunkokushugi-teki sensei seiji 軍国主義的専制 政治) ainsi que la libre circulation maritime. Les idées de Wilson, proclama Yoshino peu après la déclaration publique du président américain devant le Congrès, "auront une influence importante sur le progrès de la civilisation après la guerre " (Yoshino 1917 : 95). Le président du parti Seiyūkai 政友会, Hara Takashi 原敬 (1856-1921), nota dans son journal intime que les "affaires internationales changeront complètement " avec la belligérance américaine $^{33}$.

Si cette entrée en guerre constitua un indice de l'énorme influence potentielle des États-Unis sur la politique internationale, la fin de la guerre en fut la confirmation. Les historiens ont souvent présenté le XIX ${ }^{e}$ siècle comme une ère de changement durant laquelle le Japon passa d'une société féodale à un État moderne d'une grande puissance économique (Gordon 2014 : 93). Tandis que les spécialistes du Japon ne mentionnent généralement pas une transformation semblable après la Première Guerre mondiale, cette première guerre totale de l'histoire eut un effet similaire à celui de l'émergence de l'impérialisme moderne au XIX ${ }^{\mathrm{e}}$ siècle au Japon. Tout comme l'arrivée du commodore américain Matthew Perry en 1853 souleva un débat de grande envergure sur la "régénération " (ishin 維新) nationale japonaise, les décideurs et experts de Tokyo se saisirent de la destruction du centre de la civilisation mondiale (l'Europe) au début du $\mathrm{xx}^{\mathrm{e}}$ siècle comme d'une opportunité pour une autre transformation intégrale ${ }^{34}$. Comme le remarque Hara Takashi en juin 1917, "pour la première fois en cinquante ans depuis la restauration [de Meiji], le temps d'un renouvellement national (kokka sasshin 国家刷新) est arrivé35 ". Le magazine bi-hebdomadaire Nihon oyobi Nihonjin 日本及日本人 (Japon et Japonais) signalait en septembre 1919 que «le mot d'ordre actuel est de tout réformer (kaizō 改造)" ("Renshi hekichō»1919: 102).

33. Hara (1981, vol. 4 : 291), entrée du 2 juin 1917.

34. Parmi les historiens japonais, Kurosawa Fumitaka est un cas peu commun car il a longtemps souligné les parallèles entre le choc de l'arrivée du commodore Perry dans la baie d'Uraga en 1853 et celui de la Première Guerre mondiale, en particulier pour l'institution militaire japonaise (Kurosawa 2000 et 2013).

35. Hara (1981, vol. 4 : 291), entrée du 2 juin 1917. 
Si donc, au Japon, le mot-clé était devenu « réforme » en 1919, le modèle principal de cette réforme fut les États-Unis. Par son irrésistible pouvoir politique, militaire et économique, la Grande-Bretagne avait été la référence première du Japon au XIX ${ }^{e}$ siècle. De même, le rôle décisif des États-Unis pour la victoire dans la Première Guerre mondiale propulsa Washington au premier plan dans la vie nationale japonaise. Dès mai 1918, l'attaché militaire américain à Tokyo affirmait : «Les Japonais commencent à réaliser que nous sommes "partout" en guerre et que nous gagnerons ${ }^{36}$ ». Tandis que les responsables politiques américains avaient sévèrement critiqué les efforts allemands de propagande au Japon durant la guerre ${ }^{37}$, en 1919 "chaque journal au Japon imprime tous les jours environ deux colonnes d'informations américaines adoptant un point de vue américain ", comme le fit observer le pédagogue John Dewey, durant une tournée à travers le pays peu après l'armistice (Dewey 1982 [1919] : 150). Cette attitude très positive envers les États-Unis ne durera pas. Mais à partir de 1919 et jusqu'en 1931, les décideurs et experts japonais façonnèrent consciemment une nouvelle trajectoire nationale en suivant ce qu'ils considéraient comme le modèle le plus puissant de ce monde d'après-guerre : la solution de Woodrow Wilson pour la démocratie, l'internationalisme et le contrôle des armes ${ }^{38}$.

Considérant l'implosion, durant la guerre, des quatre belligérants principaux ayant un régime autocratique, le populaire mensuel japonais $C h \bar{u} \bar{o}$ kōron 中央公論 notait en 1918 que "le mouvement de la pensée mondiale a fait remarquablement progresser le pouvoir des partis politiques» ("Hara naikaku o mukau » $1918: 1$ ). En effet, alors que le père de la Constitution japonaise moderne, Itō Hirobumi 伊藤博文 (1841-1909), n’avait pas envisagé au départ que les partis politiques seraient au centre du régime politique japonais, le suffrage universel masculin fut introduit au Japon en 1925 tandis qu'une série de cabinets formés par des partis politiques furent au pouvoir entre 1924 et 1932. Comme Saitō Takao 斎藤隆夫 (1870-1949), député du parti Kenseikai 憲政会, le nota durant le débat sur le suffrage

36. Capitaine K. F. Baldwin, "American News in Japan", 23 mai 1918 (WDGS/ WDSS, Folder: 003011-001-0002).

37. K. F. Baldwin, "Propaganda in Japan ", 4 mars 1918 (WDGS/WDSS, Folder: 003011-001-0002).

38. Pour une étude complète sur cette entreprise, voir Dickinson (2013). 
universel masculin, "il existe des exemples de [dirigeants] détruisant des pays par leur mépris des droits et des libertés du peuple. Mais il n’existe aucun exemple de [dirigeant] détruisant un pays par son respect des droits du peuple et par le fait qu'il lui accorde la libertée $"$.

Au début du $\mathrm{xx}^{\mathrm{e}}$ siècle, le Japon impérial rejoignit les grandes puissances dans une compétition acharnée pour les territoires coloniaux. En 1919, cette compétition en était arrivé à être considérée comme une cause majeure dans le déclenchement des guerres. En tant que membre éminent de la délégation japonaise à la conférence de paix de Paris, le prince Konoe Fumimaro 近衛文麿 (1891-1945) remarqua en juin 1919 que la notion d'autodétermination soutenue par Woodrow Wilson avait acquis une place essentielle dans les débats et que l'idée d'une association au sein de laquelle les États coopéreraient, une Société des Nations, contribuerait à faire en sorte que le nom du président américain «brille éternellement dans l'histoire de l'humanitéto ». Entre 1919 et 1931, le Japon participa avec enthousiasme à un nombre remarquable de conventions internationales - le traité de Versailles, le pacte de la Société des Nations, le traité naval de Washington, le traité des Neuf Puissances, le traité des Quatre Puissances, le pacte Briand-Kellogg, le traité naval de Londres - qui constituèrent ce que les contemporains décrivirent comme une nouvelle ère de «diplomatie de conférence » ("Kaigi gaikō no ryūkō » 1922: 112).

Comme l'expansion coloniale, la course effrénée aux armements d'avant 1914 fut perçue par les réformateurs d'après-guerre comme une des causes majeures du conflit. D'après l'orateur du parti Kenseikai, Ozaki Yukio 尾崎行雄 (1858-1954), la tentative d'avant-guerre de maintenir une stabilité grâce à la puissance des armes n'entraîna qu'un "malheur inimaginable» (sōzō dekinai hodo no sanjō 想像できないほどの惨状 ; Ozaki 1921 : 5). Parmi les conventions internationales d'importance signées par le Japon dans l'entre-deux-guerres se trouve une série d'accords initiés par une invitation américaine à des pourparlers sur le désarmement, à Washington. Les

39. Ozaki Yukio, «Kokumin tanen no shukubō yōyaku mukuiraru " 国民多年の 宿望ようやく向くいらる (Les souhaits de longue date de la Nation enfin réalisés), Yomiuri shinbun 読売新聞, 3 mars 1925 ; réimprimé dans Nakajima (1978, vol. 13 : p. 95).

40. Konoe (1981 : 36-37). Ce passage est daté de juin 1919 dans un volume initialement publié en 1920 . 
échanges qu'entretinrent les Japonais avec les Américains et les Britanniques à Washington (1921-22), à Genève (1927) et à Londres (1930) réduisirent drastiquement la place des armes dans la vie japonaise. Tokyo renonça à son programme de construction de dix navires capitaux pour la Marine impériale (Evans \& Peattie 1997 : 197) et renonça également à quatre divisions de l'Armée impériale. De plus, les cabinets successifs constitués par des partis politiques réduiront les dépenses militaires de 65,4\% du budget national en 1922 à seulement 30,4\% en 1932 (Nakamura 1983 : 39).

Outre l'intégration sans précédent de l'économie japonaise avec celle des États-Unis, cette nouvelle inclination politique envers Washington fut décrite aux États-Unis comme l'aube d'un temps nouveau. Même avant l'armistice, le lieutenant-colonel K. F. Baldwin, l'attaché militaire américain au Japon, nota que Woodrow Wilson y bénéficiait d'une couverture médiatique "des plus favorables ». «Les Japonais, déclara Baldwin d'un air approbateur, semblent promouvoir une paix juste et durable ${ }^{41} »$. Avec la fin des hostilités, Baldwin salua avec enthousiasme les remarquables commémorations de l'armistice au Japon :

Après la grande victoire, une véritable célébration a eu lieu à Tokyo. Des milliers de porte-drapeaux ont visité chaque ambassade et légation ainsi que le Palais durant le jour, tandis que des dizaines de milliers de porteurs de lanternes ont fait de même la nuit. Le 21, je suis demeuré debout pendant près de six heures à l'ambassade américaine, examinant les foules qui marchaient en colonne par quatre paradant avec des drapeaux, des lanternes, des chars et des pancartes en tout genre sur lesquels on célébrait la victoire et on humiliait le kaiser. Yokohoma ainsi que d'autres villes suivront. Le maire de Tokyo a reçu deux mille cinq cents invités - des visites diplomatiques, mais pas seulement - et, devant une foule de quatre-vingt mille personnes, a exprimé ses félicitations à tous pour cette formidable victoire ${ }^{42}$.

Le nouveau sentiment de bonne entente entre le Japon et les États-Unis - que la guerre fit émerger - fut d'une importance particulière aux yeux de l'attaché militaire américain. Il observa que "les orateurs les plus éminents, tout comme la presse, font l'éloge des États-Unis en particulier ».

41. K. F. Baldwin, "Japanese Peace Terms ", 4 nov. 1918, p. 2 (WDGS/WDSS, Folder: 003011-001-0002).

42. K. F. Baldwin, "Japanese reception of the signing of the Armistice, Political ", 27 nov. 1918, p. 1 (WDGS/WDSS, Folder: 003011-001-0002). 
Ces éloges étaient fondés, à son avis, sur deux affinités bilatérales, nouvelles et fondamentales. D'un côté, les deux nations avaient maintenant des liens économiques robustes. "Il y a sans aucun doute, affirma Baldwin, un désir d'amitié plus étroite avec les États-Unis parmi les classes commerçantes ». De l'autre, le lieutenant-colonel mit l'accent sur de nouveaux intérêts politiques communs. "La presse et les orateurs publics s'intéressent vivement à l'idée d'une Société des Nations ${ }^{43}$ ». Comme John Dewey l'affirma quelques mois plus tard : «Le fait le plus impressionnant est que cette guerre a porté à la faction militariste et bureaucratique le coup le plus dur qu'elle ait jamais subi... La cause du libéralisme au Japon a fait un bond majeur en avant » (Dewey 1982 [1919] : 153-154).

\section{Conclusion}

L'histoire des tensions entre les États-Unis et le Japon, dont le point culminant fut Pearl Harbor, a fortement marqué l'analyse des relations nippoaméricaines durant la Première Guerre mondiale. Les années 1914-1918 marquèrent une ère d'expansion mutuelle, aux niveaux économique et géopolitique, produisant naturellement des tensions bilatérales. Mais dans notre hâte d'expliquer le chemin menant vers la guerre du Pacifique, nous avons négligé d'importantes synergies économiques et politiques qui, non seulement inaugurèrent une nouvelle relation bilatérale, mais formèrent également les bases d'un $\mathrm{xx}^{\mathrm{e}}$ siècle radicalement différent ${ }^{44}$.

Le saisissant repli sur soi de la puissance européenne durant la Première Guerre mondiale ouvrit la voie à de nouveaux liens économiques entre les États-Unis et le Japon, garantissant une croissance significative de leurs économies. La participation active du Japon à la paix conduite par les ÉtatsUnis après 1918 eut pour conséquence d'ajouter, en fin de compte, une base structurelle cruciale pour une coopération politique bilatérale durant

43. Ibid., p. 1-2.

44. Ce n'est que récemment que les historiens japonais ont commencé à considérer que cette guerre était non seulement aux fondements d'une ère de guerre totale, mais également d'un nouveau siècle et, plus largement, du Japon actuel. Voir Inoue (2014) et Yamamuro (2014). 
les années 1920. Si nous choisissons 1941 comme point final d'un récit portant sur les relations entre les États-Unis et le Japon, notre attention se tourne naturellement en direction des très nombreux problèmes soulevés par l'ascension respective de ces deux puissances durant la Première Guerre mondiale. Si, en revanche, nous projetons notre regard au-delà de ce désastreux milieu du Xxe siècle, il nous faut admettre que les années 1914 à 1918 ont apporté des changements fondamentaux dont le rôle fut moins de provoquer la guerre suivante que d'inaugurer un siècle nouveau. La nouvelle interdépendance économique et politique entre les États-Unis et le Japon durant la Grande Guerre et l'entre-deux-guerres est cruciale, non pas parce qu'elle mena inexorablement vers une autre guerre totale, mais parce qu'elle produisit un changement du centre de gravité géopolitique, de l'Europe vers la région Asie-Pacifique - un changement qui définit de plus en plus le monde d'aujourd'hui.

Traduit par Jean-Philippe Miller-Tremblay 


\section{Bibliographie}

\section{Sources archivistiques}

WDGS/WDSS

Records of the War Department General and Special Staffs (Record Group 165): Military Intelligence Division Files, Japan, National Archives, Washington D.C.

SKHP

Stanley K. Hornbeck Papers, Hoover Institution on War, Revolution, and Peace, Stanford.

\section{Sources publiées}

\section{ARAKI Eiko 荒木英子 2014}

Naichingēru no matsueitachi : " kango » kara yominaosu daiichiji sekai taisen ナイチンゲールの末裔たち : 〈看護〉から 読みなおす第一次世界大戦

(Les descendants de Nightingale : la Grande Guerre reconsidérée du point de vue des infirmières), Tokyo, Iwanami shoten 岩波書店.

\section{ASHER David L. 2002}

Convergence and its Costs: The Failure of Japanese Economic Reform and the Breakdown of the Washington System, 1918-1932, thèse de doctorat, University of Oxford.

\section{BEASLEY William G. 1987}

Japanese Imperialism 1894-1945, Oxford, Clarendon Press.

\section{BEERS Burton F. 1962}

Vain Endeavor: Robert Lansing's Attempt to End the American-Japanese Rivalry, Durham, NC, Duke University Press.

DEWEY John 1982 [1919]

"Japan and America ", in Jo Ann Boydson (ed.), The Middle Works of John Dewey, 1899-1924, vol. 11, Carbondale, Southern Illinois University Press: 150-155.

\section{DICKINSON Frederick R. 1999}

War and National Reinvention: Japan in the Great War, 1914-1919, Cambridge, Harvard University Asia Center.

DICKINSON Frederick R. 2003

« Japan », in Richard F. Hamilton \& Holger H. Herwig (eds.), The Origins of World War l, Cambridge, Cambridge University Press : 300-36.

DICKINSON Frederick R., 2013 World War I and the Triumph of a New Japan, 1919 - 1930, Cambridge, Cambridge University Press.

EGUCHI Keiichi 江口圭一 1989

Futatsu no taisen 二つの大戦 (Les deux Grandes Guerres), Tokyo, Shōgakukan 小学館.

\section{ELLEMAN Bruce A. 2002}

Wilson and China: $A$ Revised History of the Shandong Question, Armonk, NY, M.E. Sharpe.

\section{ESTHUS Raymond A. 1967}

Theodore Roosevelt and Japan, Seattle, University of Washington Press. 
EVANS David C.

\& PEATTIE Mark R. 1997

Kaigun: Strategy, Tactics, and Technology in the Imperial Japanese Navy, 18871941, Annapolis, Naval Institute Press.

FIFIELD Russell H. 1965

Woodrow Wilson and the Far East: The Diplomacy of the Shantung Question, Hamden, CT, Archon Books.

FISCHER Fritz 1961

Griff nach der Weltmacht. Die Kriegszielpolitik des kaiserlichen Deutschland 1914-1918, Düsseldorf, Droste Verlag.

\section{FRIEDEN Jeffrey A. 2007}

Global Capitalism: Its Fall and Rise in the Twentieth Century, New York, W. W. Norton \& Company.

GORDON Andrew 2014

A Modern History of Japan: From

Tokugawa Times to the Present, $3^{\mathrm{e}}$ éd., New York, Oxford University Press.

\section{GRISWOLD A. Whitney 1938}

The Far Eastern Policy of the United States, New York, Harcourt, Brace and Co.

HARA Keiichirō 原敬一郎 (éd.) 1981 Hara Takashi nikki 原敬日記 (Journal de Hara Takashi), 6 vol., Tokyo, Fukumura shuppan 福村出版.

« Hara naikaku o mukau » 1918

原内閣を向かう (L'arrivée du gouvernement Hara), Chūō kōron 中央 公論, 33 (10).
HAYAMI Akira 速水融 \& KOJIMA Miyoko 小嶋美代子 2004 Taishō demogurafi. Rekishi jinkōgaku de mita hazama no jidai 大正デモグラフィー 歴史人口学でみた狭間の時代 (La démographie Taishō : une époque intermédiaire considérée du point de vue de la démographie historique), Tokyo, Bunshun shinsho 文春新書.

\section{HIROBE Izumi 2001}

Japanese Pride, American Prejudice: Modifying the Exclusion Clause of the 1924 Immigration Act, Stanford, Stanford University Press.

INOUE Toshikazu 井上寿一 2014 Daiichiji sekai taisen to Nihon 第一次世界 大戦と日本 (Le Japon et la Première Guerre mondiale), Tokyo, Kōdansha 講談社.

\section{IRIYE Akira 1992}

Across the Pacific: An Inner History of American-East Asian Relations, rev. ed., Chicago, Imprint Publications.

\section{ISRAEL Jerry 1971}

Progressivism and the Open Door: America and China, 1905-1921, Pittsburgh, University of Pittsburgh Press.

ITŌ Yukio 伊藤之雄 1999

Rikken kokka no kakuritsu to Itō Hirobumi 立憲国家の確立と伊藤博文 (Itō Hirobumi et la construction d'un État constitutionnel), Tokyo, Yoshikawa kōbunkan 吉川弘文館. 
« Kaigi gaikō no ryūkō » 1922

会議外交の流行 (La vogue de la diplomatie de conférence), Kokusai chishiki 国際知識, 2 (11).

KANE Robert G. 2010

« Race and Representation: Japan and the Limits of a Wilsonian Democratic

Peace », White House Studies, 10 (4).

KAWAMURA Noriko 2000

Turbulence in the Pacific: Japanese-U.S. Relations during World War I, Westport, Praeger.

\section{KENNEDY Ross A. 2009}

The Will to Believe: Woodrow Wilson, World War I, and America's Strategy for Peace and Security, Kent, Kent State University Press.

\section{KONOE Fumimaro 近衛文麿} 1981 [1920]

Sengo Ōbei kenbunroku 戦後欧米見聞録 (Observations sur l'Europe et les ÉtatsUnis d'après-guerre), Tokyo, Chūō kōronsha 中央公論社.

KUROSAWA Fumitaka 黒沢文貴 2000 Taisenkanki no Nihon rikugun 大戦間期の 日本陸軍 (L'Armée japonaise durant l'entre-deux-guerres), Tokyo, Misuzu shobō みすず書房.

\section{KUROSAWA Fumitaka 2013}

Futatsu no «kaikoku » to Nihon 二つの 「開国」と日本 (Les deux ouvertures du Japon), Tokyo, Tokyo daigaku shuppankai 東京大学出版会.

\section{LAFEBER Walter 1997}

The Clash: U.S.-Japan Relations

Throughout History, New York, W. W. Norton.

McCLAIN James L. 2002

Japan. A Modern History, New York, W. W. Norton.

\section{MATSUDAIRA Tsuneo 1925}

"Some Principles of Japanese Foreign Policy ", Advocate of Peace through Justice, 87 (7).

\section{MORLEY James W. 1957}

The Japanese Thrust into Siberia, New York, Columbia University Press.

NAKAJIMA Kenzō 中島健蔵 (éd.) 1978 Shinbun shūroku Taishōshi 新聞集録 大正史 (L'histoire de Taishō par les sources journalistiques), 15 vol., Tokyo, Taishō shuppan 大正出版.

\section{NAKAMURA Takafusa 1983}

Economic Growth in Prewar Japan, trad. Robert A. Feldman, New Haven, Yale University Press.

[éd. or. Senzenki Nihon keizai seichō no bunseki 戦前期日本経済成長の分析，Tokyo， Iwanami shoten, 1971].

NARAOKA Sōchi 奈良岡聰智 2015 Taika nijūikka-jō yōkyū to wa nan datta no ka. Dai-ichiji sekai taisen to Nitchū tairitsu no genten 対華二十一力条要求とは 何だったのか一第一次世界大戦と日中 対立の原点 (Que furent les Vingt-et-une demandes à la Chine ? À l'origine de la confrontation sino-japonaise durant la Première Guerre mondiale), Nagoya, 
Nagoya daigaku shuppankai 名古屋 大学出版会.

NISHIDA Toshihiro 西田敏宏 2002 «Washinton taisei to Shidehara gaikō » ワシントン体制と幣原外交 (La politique extérieure de Shidehara et le système de Washington), in Itō Yukio 伊藤之雄 \& Kawada Minoru 川田稔 (éd.), Nijū seiki Nichi-Bei kankei to Higashi Ajia 20世紀 日米関係と東アジア (Les relations nippo-américanes et l'Asie orientale au $x x^{\mathrm{e}}$ siècle), Tokyo, Fūbōsha 風媒社.

\section{OHKAWA Kazushi}

\& SHINOHARA Miyohei (dir.) 1979

Patterns of Japanese Economic

Development: A Quantitative Appraisal, New Haven, Yale University Press.

\section{OKAMOTO Shumpei 1970}

The Japanese Oligarchy \& the RussoJapanese War, New York, Columbia University Press.

ŌYAMA Azusa 大山梓 (éd.) 1966

Yamagata Aritomo ikensho 山県有朋 意見書, Tokyo, Hara shobō 原書房.

\section{OZAKI Yukio 尾崎行雄 1921}

«Kokka no sonbō to kokusai renmei » 国家の存亡と国際連盟 (Survie de l'État et S.D.N.), Kokusai renmei 国際連盟, 1 (2).

« Renshi hekichō » 1919

策視壁聴 (Propos obtenus en secret), Nihon oyobi Nihonjin 日本及日本人， no. 765,15 sept.

RICHARD Carl J. 2013

When the United States Invaded Russia:
Woodrow Wilson's Siberian Disaster, Lanham (Maryland), Rowman \& Littlefield.

SAFFORD Jeffrey J. 1970

« Experiment in Containment: The United States Steel Embargo and Japan, 1917-1918 », Pacific Historical Review, 39 (4).

\section{ROOSEVELT Theodore 1920}

What the Japanese Stood for in the World War, éd. bilingue, introduction de Shibusawa Eiichi, s.l.

\section{SHIMAZU Naoko 1998}

Japan, Race and Equality: The Racial Equality Proposal of 1919, London, Routledge.

\section{SUETAKE Yoshiya 季武嘉也 1998}

Taishōki no seiji kōzō 大正期の政治構造 (La structure politique de l'ère Taishō), Tokyo, Yoshikawa kōbunkan 吉川弘文館.

TAKAHARA Shusuke 高原秀介 1997 "Uiruson seiken to "Ishii-Ranshingu kyōtei" no seiritsu » ウイルソン政権と 「石井・ランシング協定」の成立 (Le gouvernement Wilson et l'accord Lansing-Ishii), Kōbe hōgaku zasshi 神戸 法学雑誌, 47 (3).

\section{TAKAHARA Shusuke 2006a} "Uddorō Uiruson seiken to Shiberia shuppei seisaku no henyō » ウッドロー・ ウィルソン政権とシベリア出兵政策の変容 (Le gouvernement de Woodrow Wilson et l'évolution des plans relatifs à l'expédition de Sibérie), in lida Shūji 飯田収治 (éd.), Seiyō sekai no rekishizōo 
motomete 西洋世界の歴史像を求めて

(Quelle histoire pour le monde occidental ?), Nishinomiya, Kansai gakuin daigaku shuppankai 関西学院 大学出版会.

\section{TAKAHARA Shusuke 2006b}

Uiruson gaikō to Nihon. Risō to genjitsu no aida 1913-1921 ウィルソン外交と日本一 理想と現実の間 1913-1921 (La politique extérieure de Wilson et le Japon. Entre idéal et réalité), Tokyo, Sōbunsha 創文社.

\section{TAKII Kazuhiro 2014}

Ito Hirobumi: Japan's First Prime Minister and Father of the Meiji Constitution, trad. Takechi Manabu, London, Routledge, [éd. or. Itō Hirobumi: Chi no seijika 伊藤 博文 : 知の政治家, Tokyo, Chūō kōron shinsha, 2010].

\section{TOGO M. 1918}

"Japan and Ships », The North American Review, 207 (748).

\section{TREAT Payson Jackson 1918}

" Japan, America and the Great War », A League of Nations, 1 (8).

\section{WILLIAMS William J. 1996}

« Josephus Daniels and the U.S. Navy's Shipbuilding Program During World War I », The Journal of Military History, 60 (1).

\section{YAMAMURO Shin.ichi 山室信一 2011}

Fukugō sensō to sōryokusen no dansō. Nihon ni totte no daiichiji sekai taisen 複合 戦争と総力戦の断層一日本にとっての第一次 世界大戦 (Aspects de la guerre complexe et de la guerre totale. La Première
Guerre mondiale pour le Japon), Tokyo, Jinbun shoin 人文書院.

\section{YAMAMURO Shin.ichi, OKADA Akeo 岡田暁生, KOSEKI Takashi 小関隆 \&} FUJIHARA Tatsushi 藤原辰史 (dir.) 2014 Gendai no kigen. Daiichiji sekai taisen 現代の起点一第一次世界大戦 (La Première Guerre mondiale. L'origine de l'époque contemporaine), 4 vol., Tokyo, Iwanami shoten 岩波書店.

\section{YOSHINO Sakuzō 吉野作造 1916a}

«Kensei no hongi o toite, sono yūshū no bi o sumasu no michi o ronzu » 憲政の 本義を説いて其有終の美を済すの道を論ず (Comment parachever la mise en œuvre du gouvernement constitutionnel en discutant de sa véritable signification), Chūō kōron 中央公論, 31 (1) : 17-114.

\section{YOSHINO Sakuzō 1916b}

«Seishinkai no Taishō ishin » 精神界の 大正維新 (La restauration Taishō dans la sphère de l'esprit), Chūō kōron 中央 公論, 31 (1).

\section{YOSHINO Sakuzō 1917}

[ss le pseudonyme de Kosen Gakujin 古川学人] «Beikoku sansen no bunmeiteki igi » 米国参戦の文明的異議 (Objection d'ordre civilisationnel à l'entrée en guerre des États-Unis), Chūō kōron 中央公論, 32 (5). 